\title{
Chair-side saliva parameters assessment and caries experience evaluation
}

\author{
Ioana Chifor ${ }^{1}$, Laura Rusu (Dascalu) ${ }^{2}$, Andrei Picos ${ }^{1}$, Radu Chifor ${ }^{1}$,
} Iulia Badea ${ }^{1}$, Corina Tisler ${ }^{3}$, Mindra Badea $^{1}$

\begin{abstract}
1) Dental Prevention Department, Faculty of Dental Medicine, Iuliu Hatieganu University of Medicine and Pharmacy, Cluj-Napoca, Romania

2) Department of Dental Materials, Faculty of Dental Medicine, Iuliu Hatieganu University of Medicine and Pharmacy, Cluj-Napoca, Romania

3) Department of Prosthodontics, Faculty of Dental Medicine, Iuliu Hatieganu University of Medicine and Pharmacy, Cluj-Napoca, Romania
\end{abstract}

DOI: $10.15386 / \mathrm{mpr}-1523$

Manuscript received: 22.07.2019

Received in revised form: 02.10.2019 Accepted: 04.11.2019

Address for correspondence: dascalu.monica@umfcluj.ro

\begin{abstract}
Introduction. Carioreceptivity-based, individualized prevention protocols allow a minimally invasive approach in many patients, in all age groups. The long-term success of such protocols depends not only on the actual caries experience of the patients, but also on the possible responses based on natural defense mechanisms. Therefore the salivary flow, the buffer capacity of stimulated and non-stimulated saliva and mostly the Streptococcus Mutans and Lactobacilli number are highly important in deciding whether a long-term remineralization therapy can be successfully applied for early stage caries lesions. Similar tests may record the the acidogenicity of dental biofilms. These risk factors can be entered into a computerbased risk assessment program (such as Cariogram).
\end{abstract}

Methodology. An extensive literature search was performed on Medline/PubMed using as key-words: Streptococcus Mutans, Lactobacilli, saliva volume, $\mathrm{pH}$ and buffer capacity. The methodology of selecting the reviewed literature and the inclusion criteria were based on articles published in the last ten years.

Results. Besides topically applied remineralization products, either in-office or at-home, changes in the diet can diminish the number of cario-active bacteria, especially Lactobacilli. Latest researches show that dentifrices, especially toothpastes containing xylitol and/or probiotics can have a significant carioprotective effect.

Conclusions. Dental caries assessment is very important for specific age groups, because most risk factors have specific consequences in different age groups. Preteen and teenage patients need more individualized caries management strategies in order to avoid the onset of new caries lesions. Root caries risk may be decreased by an intensive and continuous preventive care.

Keywords: dental caries, dentifrices, saliva, risk factors

Carioreceptivity-based, individualized

\section{Introduction}

Standard caries experience evaluation includes: dental examination, DMF-T, DMF-S index, ICDAS, but these show the past caries experience. Nowadays caries diagnosis can be performed on molecular level by saliva and bacterial plaque tests in order to assess: Streptococcus Mutans, Lactobacilli, saliva volume, $\mathrm{pH}$ and buffer capacity. The results may predict future caries experience and allow an evidence-based design of the intensity of individualized preventive dental care. ntive care plans allow a minimally invasive approach in many patients, in all age groups. The long-term success of such protocols depends not only on the actual caries experience of the patients, but also on the possible responses based on natural defense mechanisms. The general factors such as diet, fluoride intake, general health and medication can significantly influence caries risk at all ages, if local factors mainly saliva parameters belong to higher protection interval.

The prevention of caries in young 
children is a high point of interest worldwide. The reduction of dental caries incidence is achieved by dental health education programs, but also by prevention programs which involve the use of agents like fluoride and xylitol [1].

Fluoride's action is based on fighting against teeth decay, reducing the demineralization of enamel and also increasing the remineralization. Xylitol is a polyol with many favorable effects on oral health, because it can not be metabolized by S. mutans but on the contrary, it diminishes their number and growth [2].

It has been demonstrated by various studies that the association between fluoride and xylitol is more efficient than using only fluoride against dental caries. Topical application of products that contain xylitol have shown its effects on the composition and saliva flow, enamel decay, bacteria and plaque development [3].

In the oral cavity there is an endless interaction between food components, bacterial species, teeth and saliva. Saliva contains cariogenic bacteria, with high implications in teeth decay. Streptococcus mutans has an important role in the initiation of tooth caries, but the evolution of the carious process is highly connected to Lactobacillus [4]. That is why most studies focus on these two bacterial species.

The aim of this research paper was to summarize how carioreceptivity-based, individualized prevention protocols allow a minimally invasive approach in many patients, in different age groups, as an overview of the long-term success of such protocols and the factors it depends on, such as the actual caries experience of the patients and the possible responses based on natural defense mechanisms.

\section{Material and methodology}

An extensive literature search was performed on Medline/PubMed using combinations of 2 of the following key-words: Streptococcus Mutans, Lactobacilli, saliva volume, $\mathrm{pH}$ and buffer capacity. The methodology of selecting the reviewed literature and the inclusion criteria were based on articles published in the last ten years.

\section{Results - current trends}

Salivary tests (Figure 1) can help the practitioner in establishing a correct diagnosis, by identifying, measuring and assessing the patient's saliva parameters. These tests can evaluate the hydration level of the organism, salivary consistency, $\mathrm{pH}$ level of resting or stimulated saliva, buffering capacity and stimulated saliva flow, also can guide the practitioner in choosing the right preventive care program, the appropriate dental materials in order to initiate changes needed in the patient's oral hygiene $[5,6,7]$.

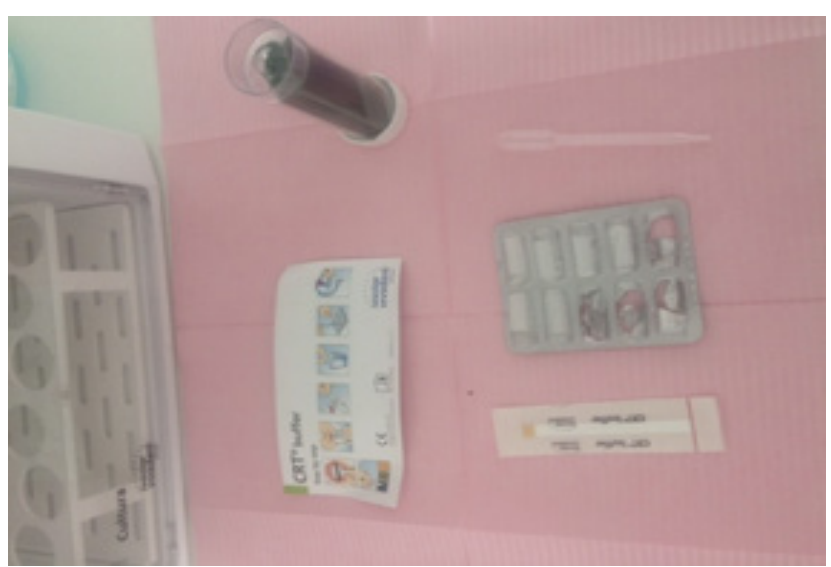

Figure 1. Chair-side saliva tests.

Educating patients to have a correct hygiene is one of the most important preventive measures and salivary tests can show the patients their oral status, stimulating a better oral care, by an interactive approach with the dental team $[8]$.

The reduced working time make salivary test ideal for usage during routine oral examinations and can determine the patients possible caries risk.

Instructions for use are easy, assessing the resting flow rate using the droplet time, collecting resting saliva, testing the $\mathrm{pH}$ of resting saliva, measuring the $\mathrm{pH}$ of the resting saliva with a paper indicator, collecting stimulated saliva into a graduated container, applying stimulated saliva to $\mathrm{pH}$ test paper using a pipette and testing buffer capacity using test pads impregnated with acid $[9,10]$.

There are three tests for assessing the resting saliva [11]. Testing the level of hydration shows the time for visible productions of saliva of the lower lip labial gland secretion (Figure 2). Values higher than 60 seconds indicates a low resting flow.

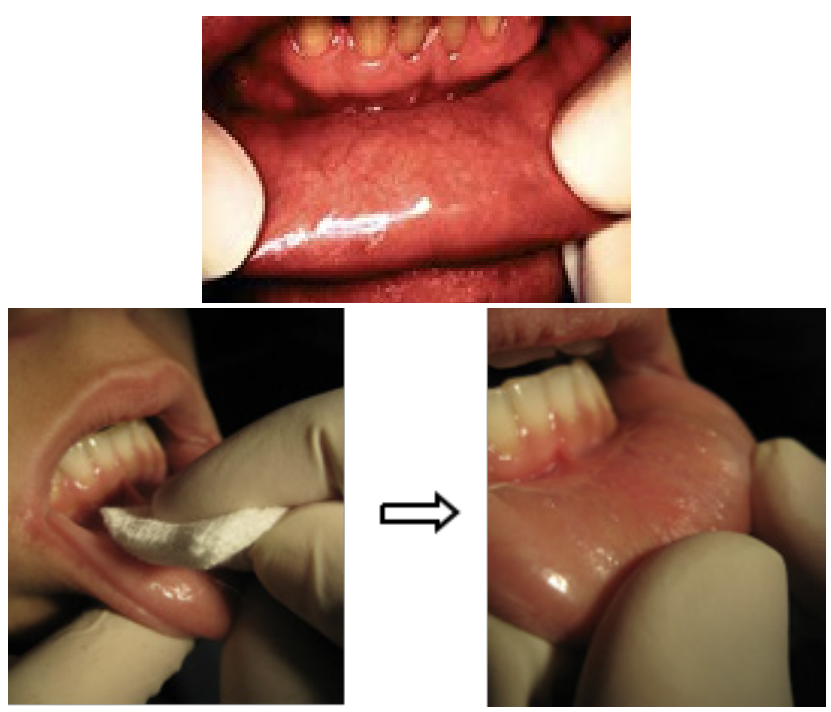

Figure 2. Testing the level of hydration. 
Saliva consistency can be observed in the oral cavity as follows: increased viscosity is represented by sticky frothy saliva residues, normal viscosity means clear and watery saliva.

The $\mathrm{pH}$ measurement (Figure 3) is made by placing the test strip into the expectorated saliva, the color indicated will show the value, 5-5.8 is highly acidic, 6-6.6 is moderately acidic and between $6.8-7.8$ is healthy saliva.
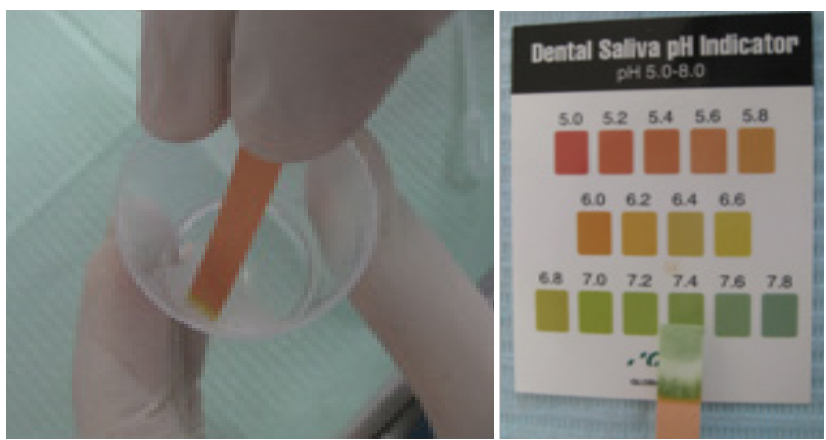

Figure 3. Testing resting saliva $\mathrm{pH}$.

Testing the stimulated saliva quantity is made after chewing tasteless wax for 5 minutes and collecting the saliva at regular intervals during this time, a normal level of flux will be above $5 \mathrm{ml}$ (Figure 4).

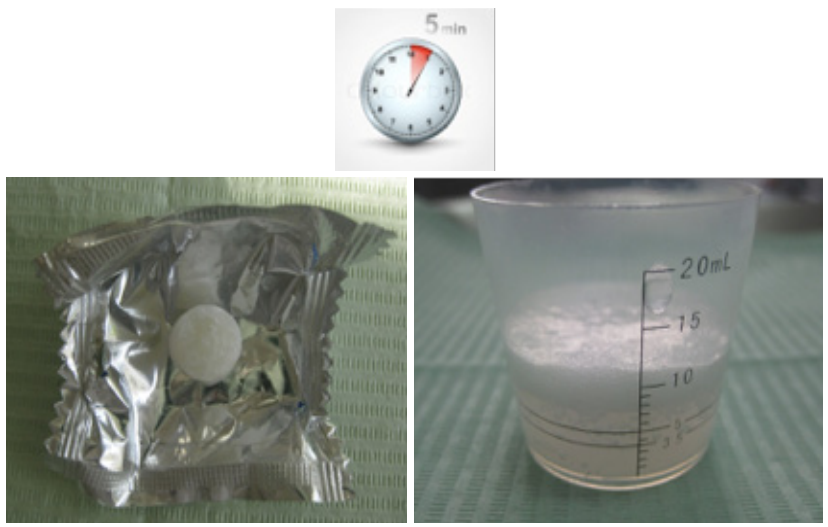

Figure 4. Collecting stimulated saliva.

Saliva tests directions of use provide clear information regarding results interpretation (Figure 5 and 6).

The buffering capacity test is made by placing a drop of saliva on each one of the three colored squares found on one strip, the color resulting afterwards has a corresponding number and the sum of the three will give the final score between 0 and 12, the value from 10 to 12 being the normal buffering capacity value (Figure 7).

\section{TESTING OF STIMULATED SALIVA}

TEST 4 - Saliva quantity

Instruct the patient to chew the piece of wax to stimulate salivary flow. After 30 seconds, let the patient expectorate into the spittoon. Continue chewing for a further 5 minutes, collecting all the saliva into the collection cup at regular intervals.

The quantity of saliva can be measured by checking the $\mathrm{mL}$ markings on the side of the cup.

Quantity of saliva at 5 minutes 2

\begin{tabular}{|c|c|}
\hline$<3.5 \mathrm{~mL}$ & Very low \\
\hline Between $5.0-3.5 \mathrm{~mL}$ & Low \\
\hline$>5.0 \mathrm{~mL}$ & Normal \\
\hline
\end{tabular}

Note:

Normal stimulated saliva flow rate may vary between $1 \mathrm{~mL} / \mathrm{min}$ $-1.6 \mathrm{~mL} / \mathrm{min}$.

Figure 5. GC stimulated saliva testing interpretation [11].

\section{TEST 5 - Buffering capacity}

a) Remove a Buffer test strip from the foil package and place onto an absorbent tissue with the test side up.

b) Using a pipette, draw sufficient saliva from the collection cup and dispense one drop onto each of the 3 test pads. Immediately turn the strip $90^{\circ}$ to soak up excess saliva on the absorbent tissue.This will prevent the excess saliva from swelling on the test pad and possibly affecting the accuracy of the test result.

c) The test pads will begin to change colour immediately and after 2 minutes the final result can be calculated by adding the points according to the final colour of each pad. See conversion table and examples underneath.

Conversion table

Test pad colour at 2 minutes

$\begin{array}{ll}\text { Green } & 4 \text { points } \\ \text { Green/Blue } & 3 \text { points* } \\ \text { Blue } & 2 \text { points } \\ \text { Red/Blue } & 1 \text { point * } \\ \text { Red } & 0 \text { points }\end{array}$

\section{Examples}

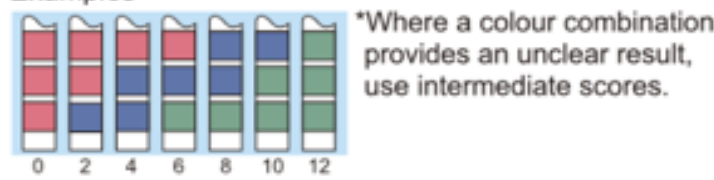

Interpreting the result. Combined total

Buffering ability of Saliva
Very low
Low
Normal / High

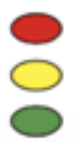

Figure 6. Saliva buffering capacity GC test [11]. 


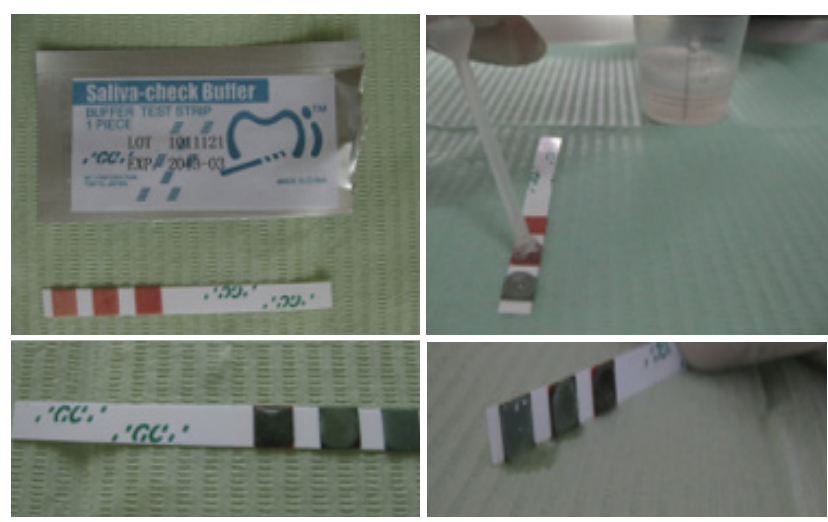

Figure 7. Stimulated saliva buffering capacity test.

Saliva characteristics are highly correlated with caries experience as it is shown also in a study performed on young, healthy adults (dental students) [12] (Table I):

Table I. DMF-S values in a group of young, healthy adults (dental students) [12].

\begin{tabular}{l|c} 
& \% of subjects \\
\hline DMF-S $\varepsilon[9-21]$ & 65 \\
DMF-S $=3$ & 2.5 \\
DMF-S $=35$ & 5
\end{tabular}

In the studied group [12] the mean DMF-S value was 18.39 , under a normal distribution. According to International Caries Assessment and Detection System examination, half of patients included in this study had no active cavities. Although almost $20 \%$ of the subjects had a $\mathrm{pH}$ within the normal interval, most of them were at the bottom value of the interval (6.8). Most subjects in this study had a moderately acidic $\mathrm{pH}$ (6.4). The mean $\mathrm{pH}$ was 6.7 , which is also a moderately acid one. The Pearson correlation coefficient between DMF-S and $\mathrm{pH}$ was 0.255 . A mild negative correlation $(-0.275)$ was found between the cariogenic food intake and buffer capacity of stimulated saliva. A week later a statistically significant decrease of cariogenic foods and drinks intakes was noticed for students with acid $\mathrm{pH}$ and with low buffer capacity. The study concluded that a regular intake of cakes, bonbons and chocolate was reported by subjects who had a high DMF-S value and a low saliva buffer capacity. Only after the dental students enrolled in the study, were aware of their caries risk, they changed their diet towards a less cariogenic one, even though they had had the theoretical knowledge regarding caries prevention for at least 3 years. Therefore the use of the chair-side salivary test could motivate cario-receptive patients.

Saliva tests for assessing the SM and LB (Figure 8) are very useful for patient education as well as for predicting the caries risk: SALIVA-CHECK SM - is a semi-quantitative- assesses if $\mathrm{S}$. mutans number is higher than $500.000 \mathrm{CFU} / \mathrm{ml}$ by immuno-chromatographymonoclonal antibodies. The results are visible in $15 \mathrm{~min}$. "Evaluation of Two Salivary Test Kits Using Speciesspecific Antibodies" by K. OHMORI, and colab. Japan IADR 2006, showed that the chair-side tests have high enough sensitivity and the results are similar to the 48-hours incubation SM and LB tests.

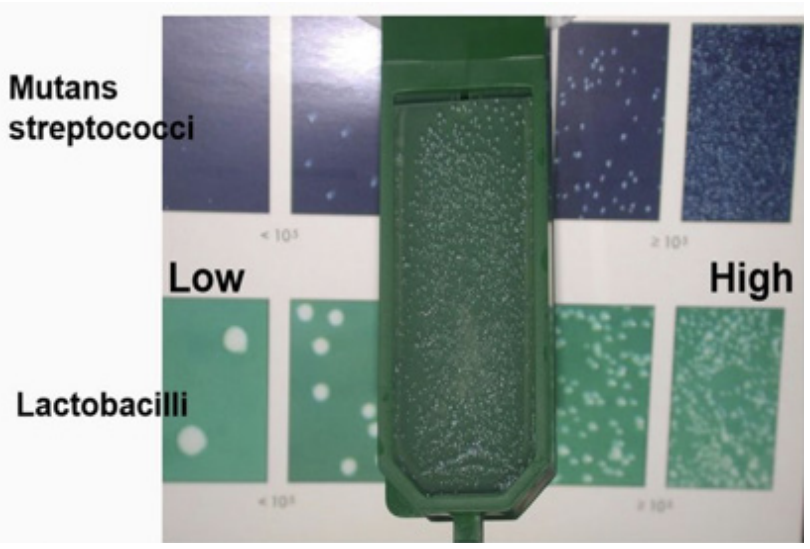

Figure 8. 48 hours incubation tests for SM and LB.

As reduced salivary flow increases acidity and the risk of caries or dental erosion, remineralisation products are indicated. Recaldent (CPP-ACP) contains Casein Phosphopeptides (CPP) and Amorphous Calcium Phosphate (ACP). Casein Phosphopeptides (CPP) are peptides derived from the milk protein, casein, that are linked to calcium $(\mathrm{Ca})$ and phosphate (PO4). In this complex, the CPP maintains/stabilizes the $\mathrm{Ca}$ and PO4 in an amorphous form (ACP) without precipitation. Recaldent (CPP-ACP) uses CPP peptides derived from the milk protein casein in order to maintain $\mathrm{Ca}$ and $\mathrm{PO} 4$ in an amorphous form (ACP). CPP will bind to surfaces such as plaque, bacteria, soft tissue and dentin, providing a reservoir of bioavailable $\mathrm{Ca}$ and $\mathrm{PO} 4$ in the saliva and at the surface of the tooth. The Amorphous Calcium Phosphate (ACP) is released from the CPP complex during oral acidic challenges. Stabilization of ACP by CPP ensures the delivery of $\mathrm{Ca}$ and $\mathrm{PO} 4$ ions into tooth structure before they precipitate/crystallize.

The first step in designing a non-invasive or minimum intervention treatment program is identifying dental caries as a disease, both in pre-cavitary and cavitary stage.

Dental caries requires a susceptible host and cariogenic bacterial plaque sustained by a high-sugar diet. The bacteria ferment the sugars, producing acid, which lowers the neutral $\mathrm{pH}$ of the oral environment and attacks the enamel. This leaches out calcium and phosphate ions, causing demineralization that leads to destruction of the enamel subsurface and the onset of dental caries, often seen as white spots. 
Varnishes are frequently used by dental practitioners because they release ions of fluoride and calcium, reduce sensitivity by sealing dentine tubules. They can be found in different flavors and do not change the color of the teeth. Usually varnishes have a retention up to few hours compared to gels or foams, that give a higher contact time between the teeth and fluoride, providing a better absorption. Because of the low viscosity, varnishes flow easily into interproximal areas as well as into pits and fissures.

Regarding caries risk, diet questionnaire should be mandatory. Sugar intake (carbohydrates) is to be assessed regarding its quantity and mostly its frequency, as well as acid drinks (quantity and frequency), time-span between meals and/or snacks. Carbohydrates $(\mathrm{CH})$ types and their physical and chemical properties (sticky foods, sugar+starch) are highly correlated with caries experience.

In the etiopathogeny of caries, genetic predisposition for dental caries is less important than the intra-familial contamination with aggressive types of SM and LB, family diet habits and oral hygiene habits [13].

Caries risk is also related to social factors like: limited access to oral hygiene products and/or limited access to dental care) due to unemployment, low education level, social habits such as alcoholism, smoking, drug addiction.

There are considered specific age group for caries risks. The first caries risk group according to the age comprises children and pre-teens during mixed dentition, due to incomplete mineralization 2 years after tooth eruption.

The next caries risk group is represented by teenagers, due to often rebel attitude, improper diet. For male teenagers $25 \%$ of calories come out of hidden sugar [14]. In adults even fully mineralized teeth may be at risk due to improper diet. The last category of caries risk is represented by elderly, with low saliva flow, exposed roots, poor diet, due to age related disabilities, general diseases and medication.

\section{Discussion}

The findings of this study are in line with recent publications $[15,16,17]$, which show that saliva, as a biological fluid can have a protective role against dental caries. The assessment methods of saliva parameters are among the most easily accessible diagnostic tools. As the above mentioned studies proved as well, it is important to follow the correct steps for saliva collection and to test immediately the sample, including the incubation for the LB and SM to start within minutes after the collection, so that the SM and LB number should not be biased by an artificial decrease due to storage at room temperature. These tests can be performed at all ages, including the cooperation of preschool children for stimulated saliva collection can be obtain, even though the accuracy of stimulated saliva volume can be influenced by the patients' cooperation. Regarding the other parameters, most researchers agree that they do not depend on the patients compliance. "Saliva diagnosis can be performed in the laboratory or directly in the dental office. Not only does the examination of the properties of this systemic secretion include the contained substances and chemical parameters, but also physical properties are increasingly explored" [15]. Thus, if saliva tests are used in the dental office, they can be associated with other easily performed chair-side tests as a diagnostic and monitoring tool in many fields of science, such as medicine, dentistry, and pharmacotherapy" [15].

Many authors highlight the importance of certain details for saliva collection protocol: "However, it should be remembered that samples of this material ought always to be collected from patients at the same time, preferably between 9:00 and 11:00 am (due to the highest physicochemical stability). A patient should not eat at least 90 minutes before the collection and stop taking medicines that affect salivary secretion 1 day before the procedure. Before sampling, the mouth should be rinsed with deionised water, and saliva should be collected for 10 minutes" [15].

\section{Conclusions}

Dental caries assessment is very important for specific age groups, because most risk factors have specific consequences in different age groups. Pre-teen and teenage patients need more individualized caries management strategies in order to avoid the onset of new caries lesions. Third age patients, as well as those suffering of xerostomia need an intensive and continuous preventive care in order to avoid root-caries. High values of SM and LB number explain caries experience of children, whereas in elderly the low buffer-capacity of saliva is directly related to cervical lesions on-set and progression. Thus the use of chair-side saliva tests could detect and motivate the carioreceptive patients, as well as those suffering of xerostomia. These chair-side saliva tests could help the clinician decide on the preventive strategies.

\section{References}

1. Chi DL, Tut O, Milgrom P. Cluster-randomized xylitol toothpaste trial for early childhood caries prevention. J Dent Child (Chic). 2014;81:27-32.

2. Sano H, Nakashima S, Songpaisan Y, Phantumvanit P. Effect of a xylitol and fluoride containing toothpaste on the remineralization of human enamel in vitro. J Oral Sci. 2007;49:67-73.

3. Salli KM, Gürsoy UK, Söderling EM, Ouwenhand AC. Effects of Xylitol and Sucrose Mint Products on Streptococcus mutans Colonization in a Dental Simulator 
Model. Curr Microbiol. 2017;74:1153-1159.

4. Maden EA, Altun C, Ozmen B, Basak F. Antimicrobial Effect of Toothpastes Containing Fluoride, Xylitol, or Xylitol-Probiotic on Salivary Streptococcus mutans and Lactobacillus in Children. Niger J Clin Pract. 2018;21:134138.

5. Lussi A, Jaeggi T. Erosion--diagnosis and risk factors. Clin Oral Investig. 2008;12 Suppl 1:S5-S13.

6. Picos A, Badea ME, Dumitrascu DL. Dental erosion in gastro-esophageal reflux disease. A systematic review. Clujul Med. 2018;91:387-390.

7. Picos AM, Chisnoiu AM, Lasserre JF, Spinei A, Chisnoiu MR, Picos A. Dental erosion - literature update. HVM Bioflux. 2013;5:135-141.

8. Lussi A, Jaeggi T, Zero D. The role of diet in the aetiology of dental erosion. Caries Res. 2004;38 Suppl 1:34-44.

9. Picos AM, D'Incau E, Bonafos C, Berar A, Chira A, Dumitrascu D. Erosion dentaire d'origine intrinseque. Revue d'odonto stomatologie. 2014;43:56-67.

10. Corrêa MC, Lerco MM, Cunha Mde L, Henry MA. Salivary parameters and teeth erosions in patients with gastroesophageal reflux disease. Arq Gastroenterol. 2012;49:214-218.
11. Saliva-Check Buffer. Available from: http://www.gcamerica. com/products/preventive/Saliva_Check_BUFFER/SalivaCheckBUFFER_8IFU.pdf

12. Chifor I, Badea I, Chifor R, Popa D, Staniste L, Tarmure $\mathrm{D}$, et al. Saliva characteristics, diet and carioreceptivity in dental students. Clujul Med. 2014;87:34-39.

13. Sharma P, Goswami M, Singh D, Massod SS, Nganba K. Correlation of Streptococcus mutans count in Mother-child Pair of Working and Nonworking Mothers: A Cross-sectional Study. Int J Clin Pediatr Dent. 2016;9:342-348.

14. Azaïs-Braesco V, Sluik D, Maillot M, Kok F, Moreno LA. A review of total \& added sugar intakes and dietary sources in Europe. Nutr J. 2017;16:6.

15. Kubala E, Strzelecka P, Grzegocka M, Lietz-Kijak D, Gronwald H, Skomro P, et al. A Review of Selected Studies That Determine the Physical and Chemical Properties of Saliva in the Field of Dental Treatment. Biomed Res Int. 2018;2018:6572381.

16. Frenkel ES, Ribbeck K. Salivary mucins in host defense and disease prevention. J Oral Microbiol. 2015;7:29759.

17. Mira A. Oral Microbiome Studies: Potential Diagnostic and Therapeutic Implications. Adv Dent Res. 2018;29:71-77. 